\title{
Study of Winter Wheat Yield Quality Analysis at ARDS Turda
}

\author{
Ovidiu Adrian CECLAN*, Ionuț RACZ, Rozalia KADAR, Adina VARADI, Diana HIRISCAU \\ Agricultural Research and Development Station, Turda, 27 Agriculturii Street,401100, \\ Cluj County, Romania \\ * corresponding author: adi.ceclan@gmail.com
}

Bulletin UASVM series Agriculture 73(2)/2016

Print ISSN 1843-5246; Electronic ISSN 1843-5386

DOI 10.15835/buasvmcn-agr: 12406

\begin{abstract}
The purpose of this research is to study the potential for yield and quality indicators for winter wheat genotypes in terms of pedological and climate condition and applied technology, at ARDS Turda during 2014 2015. Depending on the climatic conditions that are associated with applied technology is a decisive factor in successful wheat crop for all genotypes that were studied at Ards Turda during the $2014-2016$. That's wy each genotype responded differently to the conditions of the ARDS Turda also through the two levels of fertilisations applied in the winter with fertilizers $20: 20: 0,250 \mathrm{~kg} / \mathrm{ha}$ assuring $50 \mathrm{~kg} / \mathrm{ha} \mathrm{N}$ and P active substance and second level of fertilisations with $150 \mathrm{~kg} / \mathrm{ha}$ ammonium nitrate assuring $50 \mathrm{~kg} / \mathrm{ha} \mathrm{N}$ active substance. All genotype that were studied in terms of yield and quality indicators were influenced by the fertilization level. The influence of pedo-climatic conditions, applied technologies and fertilizers level at ARDS Turda showed that all genotypes with small yield had higher protein and gluten content respectively Zeleny index.
\end{abstract}

Keywords: climate condition, quality, winter wheat, yield.

\section{INTRODUCTION}

Wheat is one of the mostimportant food plants, food accounted for 35-40 \% of world population. Wheat quality is phenotypic expression of genotype interaction with environmental conditions and crop technology (Săulescu, 1984; Kadar et al., 1999, 2002). The value of a variety of winter wheat is given by its high capacity production associated with the best quality, but a very important factor is the stability of production especially in terms of adverse climatic factors (Racz et al., 2014). Choosing genotypes depending on the area or climatic conditions associated with applied technology are critical factors in the success of the wheat crop both in terms of production and quality (Ceclan et al., 2015)

Production capacity is a complex and determined by intrinsic factors or external factors influence (Ceapoiu, 1974, 1984). The main means of obtaining high stable yields is also the most dynamic factor in the use of crop varieties and hybrids of high productivity (Ceapoiu et al., 1984).

\section{MATERIALS AND METHODS}

The research was conducted during 2014 2015 in the experimental field of the Winter wheat breeding Laboratory from ARDS Turda on soil type mold cambic, vertic (faeoziom S.R.T.S. 2003) with a succession of horizons $\mathrm{Am} \rightarrow \mathrm{Bvy} \rightarrow \mathrm{C}$ or $\mathrm{Cca}$ silty loam / clay, hard, made against a background of clay marl lithology inflated with an external drain very slowly and low permeability. The ARDS Turda is situated in the north-east of Turda, $3 \mathrm{~km}$ from the highway E60 and $30 \mathrm{~km}$ from the city of Cluj Napoca. The thermal regime is characterized by average annual air of $8.6^{\circ} \mathrm{C}$, the warmest being July with average monthly temperature of $19.3^{\circ} \mathrm{C}$, while the coldest month is January with average monthly temperature $-4.4^{\circ}$ C. Average annual rainfall of $556 \mathrm{~mm}$, being the wettest on June, when $80.6 \mathrm{~mm}$ fell on average and the driest in February when it has averaged only $18.2 \mathrm{~mm}$.

Experience with winter wheat genotypes from ARDS Turda included a wide range of Romanian and foreign genotypes created in the following centers: 
ARDS Turda: Arieşan (var. ferrugineum), APULLUM (erythrospermum), TURDA 2000 (erythrospermum), DUMBRAVA (erythrospermum), ANDRADA (var. ferrugineum, fig. 4);

INCDA Fundulea: DELABRAD (eryth.), FAUR (eryth.),GLOSA (eryth), MIRANDA (eryth.), PITAR (eryth.);

Oradea: CRIŞANA (eryth.);

ARDS Suceava: MAGISTRAL (eryth.);

ARDS Lovrin: LOVRIN 34 (eryth.);

Austria: CAPO (eryth.), JOSEF (eryth.); GALIO (eryth.), FULVIO (eryth);

Hungarya: BERES (eryth.); MV. KOLO (eryth.);

France: RENAN (eryth.), APACHE (lutescens), ARLEQUIN (lutescens), Cristina (eryth.), Element (eryth.);

Germany (KWS): EXOTIC (eryth.)

Before sowing wheat grains were treated with Dividend $1 \mathrm{l} /$ tonn, against seed-borne diseases such as Tilletia spp. and Fusarium. Sowing was done individually for each variant in plots of $7 \mathrm{~m}^{2}$ and the harvestable of $5 \mathrm{~m}^{2}$. Fertilization was performed in two steps: În first were applied fertilizer 20: 20: 0 on all plots at a dose of $250 \mathrm{~kg} / \mathrm{ha}$ to provide $50 \mathrm{~kg} / \mathrm{ha}$ nitrogen and phosphorus active substance in winter on frozen on December 8. 2014. În the second step were applied to the supplementary fertilization $150 \mathrm{~kg} / \mathrm{ha}$ of ammonium nitrate, to provide 50 $\mathrm{kg} /$ ha nitrogen active substance. The statistical processing of the experimental results was done by analysis of variance based on Fisher criterion (sample F) and Duncan test. It was determined the protein content, the wet gluten and Zeleny index performed by using INFRAGRAIN 9500 analyzer.

\section{RESULTS AND DISCUSSION}

Tab. 1 presents the analysis of variance in bifactorial experience $(2 \times 25)$ placed after the subdivided parcels method in three repetitions, is found by applying the criterion of Fisher that among the factors studied, the greatest influence on the yield of winter wheat has fertilization $(\mathrm{F}=284,247)$, followed by genetic factor $(\mathrm{M}=$ 128.662) is important to mention FxS interaction $(\mathrm{F}=2.019)$.

In Tab. 2 shows the influence of fertilization on the production of winter wheat varieties such variant that additional nitrogen was applied yielded an average production of $893 \mathrm{~kg} /$ ha higher than the version N50P50.
The first three places after the yields obtained lies in this order: Apache (France) Dumbrava (Romania) Exotic (Germany). În this experience were taken into account differences from two witnesses: Arieşan variety and average yields of all varieties (Tab. 3 ).

The winter wheat genotypes react differently to fertilization, the yield increases between 403 and $1565 \mathrm{~kg} / \mathrm{ha}$ (Tab. 4), noting among these kinds Dumbrava which were obtained $10060 \mathrm{~kg} /$ ha in variant $\mathrm{N} 1100 \mathrm{~kg} / \mathrm{ha} \mathrm{N}$ to and $8778 \mathrm{k} / \mathrm{ha}$ in variant $\mathrm{N} 2$ to $50 \mathrm{~kg} / \mathrm{ha} \mathrm{N}$ active substance.

Year of 2015 was favorable for the manifestation of the production potential of winter wheat varieties tested, but was weak in terms of the quality of the crops in almost all varieties. Analysis of variance for protein content (Tab. 5) indicates that this index assessing the quality of wheat is largely influenced by genotype and And the sample F shown indicates that there are genetic differences between the varieties in terms of protein content.

Fertilization, especially with nitrogen contribute significantly to achieving good quality for grain, containing appropriate protein for baking industry. În 2015 at ARDS Turda was obtained from the average protein content of $9.51 \%$ based on the first level of fertilizer, which increases to $10.23 \%$ at the dose of $100 \mathrm{~kg} / \mathrm{ha} \mathrm{N}$ active substance (Tab. 6).

Protein content varied between $10.70 \%$ (MV. Kolo) and 8.83\% (Arlequin) and Duncan test allowed a classification of varieties of this view in six graded as follows:

Class I (a): Mv Kolo, Cristina and Ariesan (10.63 to 10.70\%);

Class II ( $\mathrm{ab}$ and $\mathrm{abc}$ ): Renan, Josef Beres, containing between 10.35 and $10.47 \%$;

Class III (abcd, bcde, cdef): Fulvio, Stone, Turda 2000 Delabrad, Element, Glos Capo (10.00 to $10.18 \%$ ); Clasa IV (defg, cdef, cde): Exotic, Crișana, Lovrin 34, Andrada, Apullum, Miranda, Galio $(9,58-9,78)$;

Class V (hi, hi): Faur and Magistral (9.33 to 9.38\%); Class VI (ij, j): Apache, Dumbrava and Arlequin.

Most varieties were classified in classes III and IV for quality (Tab. 7), having a protein content of between 9.58 and $10.18 \%$.

The results obtained from the Arieșan variety the protein content in 2015 averaged $11.47 \%$ in additional variant fertilized with nitrogen and $9.80 \%$ in the variant N50 (Tab. 8). The reaction to the fertilizer varieties of winter wheat tested in 
Tab. 1. Analysis of variance and $F$ sample for the polifactorial experience $(2 x 25)$ with winter wheat varieties of for grain yield (q / ha), at ARDS Turda, 2015

\begin{tabular}{lccccc}
\hline Variation source & Square sum & GL & Square mean & F & Signf. \\
\hline Fertilization (F) & 2991,149 & 1 & 2991,149 & 284,257 & $* * *$ \\
\hline Genotipe (G) & 3075,196 & 24 & 128,133 & 128,662 & $* * *$ \\
\hline Fx G & 315,775 & 24 & 13,157 & 2,019 & $* *$ \\
\hline Repetions (R) & 20,043 & 2 & 10,021 & & \\
\hline FxR & 21,045 & 2 & 10,522 & & \\
\hline GxR & 339,894 & 48 & 7,081 & & \\
\hline FxGxR & 285,721 & 48 & 5,952 & & \\
\hline Erorr F & 21,045 & 2 & 10,522 & & \\
\hline Erorr G & 625,615 & 96 & 6,516 & & \\
\hline Total & 7048,824 & 149 & &
\end{tabular}

Tab. 2. Influence of fertilization on yield (q / ha) for winter wheat varieties at ARDS Turda, 2015

\begin{tabular}{lcccccc}
\hline Variant & Symbol & $\begin{array}{c}\text { Yield } \\
(\mathrm{q} / \mathrm{ha})\end{array}$ & Yield (\%) & Diff to F1 (q/ha) & Signif. & Duncan \\
\hline N100P50 & F1 & 90,72 & 100,0 & 0,00 & Mt. & a \\
\hline N50P50 & F2 & 81,79 & 90,2 & $-8,93$ & 00 & b \\
\hline LSD p 5\% & & DS 5\% & & 2,28 & 2,28 \\
\hline LSD p 1\% & & & 5,26 & \\
\hline LSD p 0,1\% & & \multicolumn{3}{r}{16,74} & \\
\hline
\end{tabular}

Tab. 3. The influence of genotype on yield (q/ha) for winter wheat varieties at ARDS Turda, 2015

\begin{tabular}{|c|c|c|c|c|c|c|}
\hline Variant & Symbol & $\begin{array}{l}\text { Yield } \\
\text { (q/ha) }\end{array}$ & $\begin{array}{l}\text { Yield (\%) } \\
\text { Genotype } \\
\text { mean }\end{array}$ & $\begin{array}{c}\text { Yield }(\%) \\
\text { S1 Arieşan }\end{array}$ & $\begin{array}{l}\text { Diff to S1 } \\
\text { (q/ha) }\end{array}$ & $\begin{array}{l}\text { Diff. to genotype } \\
\text { mean (q/ha) }\end{array}$ \\
\hline ARIESAN & $\mathrm{S} 1$ & 86.74 & 100.6 & 100.0 & $0.00(\mathrm{Mt})$ & 0.48 \\
\hline APULLUM & $\mathrm{S} 2$ & 83.35 & 96.6 & 96.1 & -3.390 & -2.91 \\
\hline TURDA 2000 & S3 & 83.96 & 97.3 & 96.8 & -2.78 & -2.29 \\
\hline DUMBRAVA & S4 & 94.19 & 109.2 & 108.6 & $7.45^{* * *}$ & 7.93*** \\
\hline ANDRADA & S5 & 87.46 & 101.4 & 100.8 & 0.72 & 1.21 \\
\hline DELABRAD & S6 & 82.04 & 95.1 & 94.6 & -4.7000 & -4.2200 \\
\hline FAUR & S7 & 89.06 & 103.3 & 102.7 & 2.32 & 2.81 \\
\hline GLOSA & S8 & 83.10 & 96.3 & 95.8 & -3.640 & -3.160 \\
\hline MIRANDA & S9 & 86.28 & 100.0 & 99.5 & -0.46 & 0.02 \\
\hline PITAR & S10 & 84.82 & 98.3 & 97.8 & -1.92 & -1.44 \\
\hline JOSEF & S11 & 82.61 & 95.8 & 95.2 & -4.1300 & -3.640 \\
\hline RENAN & S12 & 84.16 & 97.6 & 97.0 & -2.58 & -2.09 \\
\hline CAPO & S13 & 84.01 & 97.4 & 96.9 & -2.73 & -2.25 \\
\hline APACHE & S14 & 95.62 & 110.9 & 110.2 & $8.88^{* * *}$ & $9.36^{* * *}$ \\
\hline ARLEQUIN & S15 & 91.35 & 105.9 & 105.3 & $4.61^{* *}$ & $5.09^{* * *}$ \\
\hline EXOTIC & S16 & 93.59 & 108.5 & 107.9 & $6.85 * * *$ & $7.34^{* * *}$ \\
\hline MV. KOLO & S17 & 79.34 & 92.0 & 91.5 & -7.40000 & -6.91000 \\
\hline CRISTINA & S18 & 78.89 & 91.5 & 90.9 & -7.85000 & -7.37000 \\
\hline ELEMENT & S19 & 85.34 & 98.9 & 98.4 & -1.41 & -0.92 \\
\hline BERES & S20 & 80.72 & 93.6 & 93.1 & -6.02000 & -5.54000 \\
\hline GALIO & S21 & 88.77 & 102.9 & 102.3 & 2.03 & 2.51 \\
\hline FULVIO & S22 & 86.63 & 100.4 & 99.9 & -0.11 & 0.38 \\
\hline MAGISTRAL & S23 & 94.20 & 109.2 & 108.6 & $7.46^{* * *}$ & $7.94^{* * *}$ \\
\hline LOVRIN 34 & S24 & 84.58 & 98.1 & 97.5 & -2.16 & -1.68 \\
\hline CRIȘANA & S25 & 85.58 & 99.2 & 98.7 & -1.16 & -0.67 \\
\hline Genotype mear & & 86.26 & 100.0 & & & $0.00(\mathrm{Mt})$ \\
\hline
\end{tabular}


Tab. 4. Influence of fertilization on yield (q/ha) for winter wheat varieties at SCDA Turda, 2015

\begin{tabular}{|c|c|c|c|c|c|c|c|c|c|c|}
\hline \multirow[b]{2}{*}{ Variant } & \multirow[b]{2}{*}{ Symbol } & \multicolumn{4}{|c|}{$\mathrm{N} 1=100 \mathrm{~kg} / \mathrm{ha} \mathrm{N}$ s.a. } & \multicolumn{4}{|c|}{$\mathrm{N} 2=50 \mathrm{~kg} / \mathrm{ha} N$ s.a. } & \multirow[b]{2}{*}{$\begin{array}{c}\text { Yield diff } \\
\text { (kg/ha) } \\
\text { N1-N2 }\end{array}$} \\
\hline & & $\begin{array}{l}\text { Yield } \\
\text { (q/ha) }\end{array}$ & $(\%)$ & $\begin{array}{c}\text { Diff. S1 } \\
\text { q/ha }\end{array}$ & Signif. & $\begin{array}{l}\text { Yield } \\
\text { (q/ } \\
\text { ha) }\end{array}$ & $(\%)$ & $\begin{array}{l}\text { Diff. } \\
\text { S1 } \\
\text { q/ha }\end{array}$ & Signif. & \\
\hline ARIESAN & S1 & 91.05 & 100.0 & 0.00 & Mt. & 82.43 & 100.0 & 0.00 & Mt. & 862 \\
\hline APULLUM & S2 & 89.61 & 98.4 & -1.44 & - & 77.09 & 93.5 & -5.34 & 0 & 1252 \\
\hline TURDA 2000 & S3 & 87.54 & 96.1 & -3.51 & - & 80.39 & 97.5 & -2.05 & - & 715 \\
\hline DUMBRAVA & S4 & 100.60 & 110.5 & 9.55 & $* * *$ & 87.78 & 106.5 & 5.35 & * & 1282 \\
\hline ANDRADA & S5 & 91.88 & 100.9 & 0.84 & - & 83.04 & 100.7 & 0.61 & - & 884 \\
\hline DELABRAD & S6 & 85.04 & 93.4 & -6.01 & 00 & 79.04 & 95.9 & -3.39 & - & 600 \\
\hline FAUR & S7 & 92.50 & 101.6 & 1.45 & - & 85.63 & 103.9 & 3.19 & - & 687 \\
\hline GLOSA & S8 & 90.92 & 99.9 & -0.12 & - & 75.27 & 91.3 & -7.16 & 000 & 1565 \\
\hline MIRANDA & S9 & 89.30 & 98.1 & -1.75 & - & 83.26 & 101.0 & 0.83 & - & 604 \\
\hline PITAR & S10 & 90.58 & 99.5 & -0.47 & - & 79.05 & 95.9 & -3.38 & - & 1153 \\
\hline JOSEF & S11 & 88.07 & 96.7 & -2.98 & - & 77.16 & 93.6 & -5.27 & 0 & 1091 \\
\hline RENAN & S12 & 88.14 & 96.8 & -2.91 & - & 80.19 & 97.3 & -2.24 & - & 795 \\
\hline CAPO & S13 & 89.21 & 98.0 & -1.83 & - & 78.80 & 95.6 & -3.63 & - & 1041 \\
\hline APACHE & S14 & 101.03 & 111.0 & 9.98 & $* * *$ & 90.21 & 109.4 & 7.78 & $* * *$ & 1109 \\
\hline ARLEQUIN & S15 & 96.94 & 106.5 & 5.90 & $* *$ & 85.76 & 104.0 & 3.32 & - & 1078 \\
\hline EXOTIC & S16 & 97.54 & 107.1 & 6.49 & $* *$ & 89.65 & 108.8 & 7.21 & $* * *$ & 789 \\
\hline MV. KOLO & S17 & 85.06 & 93.4 & -5.99 & 00 & 73.63 & 89.3 & -8.80 & 000 & 1143 \\
\hline CRISTINA & S18 & 83.09 & 91.3 & -7.96 & 000 & 74.69 & 90.6 & -7.74 & 000 & 840 \\
\hline ELEMENT & S19 & 87.63 & 96.3 & -3.41 & - & 83.04 & 100.7 & 0.60 & - & 459 \\
\hline BERES & S20 & 82.76 & 90.9 & -8.29 & 000 & 78.68 & 95.4 & -3.75 & - & 408 \\
\hline GALIO & S21 & 94.08 & 103.3 & 3.03 & - & 83.46 & 101.2 & 1.03 & - & 1062 \\
\hline FULVIO & S22 & 88.65 & 97.4 & -2.40 & - & 84.62 & 102.7 & 2.19 & - & 403 \\
\hline MAGISTRAL & S23 & 97.39 & 107.0 & 6.35 & ** & 91.00 & 110.4 & 8.57 & $* * *$ & 639 \\
\hline LOVRIN 34 & S24 & 90.15 & 99.0 & -0.90 & - & 79.01 & 95.8 & -3.42 & - & 1114 \\
\hline CRIȘANA & S25 & 89.30 & 98.1 & -1.75 & - & 81.87 & 99.3 & -0.57 & - & 743 \\
\hline
\end{tabular}

Tab. 5. Analysis of variance and $\mathrm{F}$ sample for the polifactorial experience $(2 \times 25)$ with winter wheat varieties of for protein (\%), at ARDS Turda, 2015

\begin{tabular}{lccccc}
\hline Variation source & Square sum & Liberty degrees & Mean Square & Proba F & Signif. \\
\hline Fertilization (F) & 19,512 & 1 & 19,512 & 192,680 & $* * *$ \\
\hline Genotipe (G) & 39,937 & 24 & 1,664 & 16,174 & $* * *$ \\
\hline F x G & 9,639 & 24 & 0,401 & 3,904 & $* *$ \\
\hline Repetions (R) & 1,253 & 2 & 0,626 & & \\
\hline FxR & 0,202 & 2 & 0,101 & & \\
\hline GxR & 3,556 & 48 & 0,074 & \\
\hline FxGxR & 6,320 & 48 & 0,131 & \\
\hline Erorr F & 0,202 & 2 & 0,101 & \\
\hline Erorr G & 9,877 & 96 & 0,102 & \\
\hline Total & 80,422 & 149 & & & \\
\hline
\end{tabular}

Tab. 6. Influence of fertilization on protein content for winter wheat varieties at ARDS Turda, 2015

\begin{tabular}{|c|c|c|c|c|c|c|}
\hline Variant & Symbol & Protein & $(\%)$ & Diff to $F_{1}(\%)$ & Signif. & Duncan \\
\hline $\mathbf{N}_{100} P_{50}$ & $\mathrm{~F}_{1}$ & 10,23 & 100,0 & 0,00 & Mt. & $\mathrm{a}$ \\
\hline $\mathbf{N}_{50} \mathbf{P}_{50}$ & $\mathrm{~F}_{2}$ & 9,51 & 92,8 & $-0,72$ & 00 & $\mathrm{~b}$ \\
\hline DL p 5\% & \multirow{3}{*}{\multicolumn{2}{|c|}{ DS 5\% }} & & 0,22 & & \multirow{3}{*}{0,22} \\
\hline DL p 1\% & & & & 0,52 & & \\
\hline DL p $0,1 \%$ & & & & 1,64 & & \\
\hline
\end{tabular}


Tab. 7. The influence of genotype to protein content (\%) for winter wheat at ARDS Turda, 2015

\begin{tabular}{|c|c|c|c|c|c|c|}
\hline Variant & Simbol & Protein & $(\%)$ & $\begin{array}{c}\text { Diff to } S_{1} \\
(\%)\end{array}$ & Signif & Duncan \\
\hline ARIESAN & $\mathrm{S}_{1}$ & 10,63 & 100.0 & 0,00 & Mt. & $\mathrm{a}$ \\
\hline APULLUM & $\mathrm{S}_{2}$ & 9,62 & 90,4 & $-1,02$ & 000 & fgh \\
\hline TURDA 2000 & $\mathrm{~S}_{3}$ & 10,08 & 94,8 & $-0,55$ & 00 & bcde \\
\hline DUMBRAVA & $\mathrm{S}_{4}$ & 8,92 & 83,9 & $-1,72$ & 000 & $\mathrm{j}$ \\
\hline ANDRADA & $\mathrm{S}_{5}$ & 9,63 & 90,6 & $-1,00$ & 000 & fgh \\
\hline DELABRAD & $\mathrm{S}_{6}$ & 10,02 & 94,2 & $-0,62$ & 00 & cdef \\
\hline FAUR & $\mathrm{S}_{7}$ & 9,38 & 88,2 & $-1,25$ & 000 & ghi \\
\hline GLOSA & $\mathrm{S}_{8}$ & 10,00 & 94,0 & $-0,63$ & 000 & cdef \\
\hline MIRANDA & $\mathrm{S}_{9}$ & 9,58 & 90,1 & $-1,05$ & 000 & fgh \\
\hline PITAR & $\mathrm{S}_{10}$ & 10,17 & 95,6 & $-0,47$ & 0 & bcd \\
\hline JOSEF & $\mathrm{S}_{11}$ & 10,40 & 97,8 & $-0,23$ & - & $\mathrm{abc}$ \\
\hline RENAN & $\mathrm{S}_{12}$ & 10,47 & 98,4 & $-0,17$ & - & $\mathrm{ab}$ \\
\hline CAPO & $\mathrm{S}_{13}$ & 10,00 & 94,0 & $-0,63$ & 000 & cdef \\
\hline APACHE & $\mathrm{S}_{14}$ & 9,02 & 84,8 & $-1,62$ & 000 & $\mathrm{ij}$ \\
\hline ARLEQUIN & $\mathrm{S}_{15}$ & 8,83 & 83,1 & $-1,80$ & 000 & $\mathrm{j}$ \\
\hline EXOTIC & $\mathrm{S}_{16}$ & 9,78 & 92,0 & $-0,85$ & 000 & defg \\
\hline MV. KOLO & $\mathrm{S}_{17}$ & 10,70 & 100,6 & 0,07 & - & $\mathrm{a}$ \\
\hline CRISTINA & $\mathrm{S}_{18}$ & 10,65 & 100,2 & 0,02 & - & $\mathrm{a}$ \\
\hline ELEMENT & $\mathrm{S}_{19}$ & 10,02 & 94,2 & $-0,62$ & 00 & cdef \\
\hline BERES & $\mathrm{S}_{20}$ & 10,35 & 97,3 & $-0,28$ & - & $\mathrm{abc}$ \\
\hline GALIO & $\mathrm{S}_{21}$ & 9,58 & 90,1 & $-1,05$ & 000 & fgh \\
\hline FULVIO & $\mathrm{S}_{22}$ & 10,18 & 95,8 & $-0,45$ & 0 & bcd \\
\hline MAGISTRAL & $\mathrm{S}_{23}$ & 9,33 & 87,9 & $-1,30$ & 000 & hi \\
\hline LOVRIN 34 & $\mathrm{~S}_{24}$ & 9,65 & 90,8 & $-0,98$ & 000 & fgh \\
\hline CRIŞANA & S25 & 9,72 & 91,4 & $-0,92$ & 000 & efgh \\
\hline DL p 5\% & DS 5\% & & & 0,37 & & \\
\hline DL p 1\% & & & & 0,49 & & \\
\hline DL p 0,1\% & & & & 0,63 & & \\
\hline
\end{tabular}

conditions of 2015 at ARDS Turda was different in terms of protein content, allowing identification of varieties very productive, as Arlequin which yielded low levels on both levels fertilization. Ariesan varieties and Glosa react best to nitrogen fertilization, yielding values of 1.67 and $1.87 \%$ higher at N100 variant compared to N50.

The results obtained in 2015 showed a strong influence on the wet gluten content of factors: fertilization and variety, and the interaction between them (Tab. 9).

By increasing the dose of nitrogen to obtain an increase in wet gluten content of winter wheat varieties in 2015 was $1.16 \%$ in variant $\mathrm{N} 1$ (100 $\mathrm{kg} /$ ha N) compared to $\mathrm{N} 2$ (50 kg/ha N), as shown by the data presented in Tab. 10 .
Using for control the variety Arieșan we observe that the wet gluten content that it was surpassed only by three genotypes, namely: Beres, Cristina and MV. Kolo (Tab. 11).

After wet gluten content values obtained the studied varieties could be grouped as follows:

$>21 \%$ Beres;

20.1-21\%: Capo, Renan, Josef, Arieşan, MV. Kolo, Cristina;

19.1-20 \%: Element, Delabrad, Turda 2000, Pitar, Fulvio, Glosa;

18.1-19 \%: Crişana, Faur, Andrada, Lovrin 34, Exotic, Apullum, Galio;

17.1-18 \%: Apache, Magistral, Miranda;

16.1-17 \%: Arlequin şi Dumbrava 
Tab. 8. Influence of fertilization on protein content (\%) for winter wheat varieties at ARDS Turda, 2015

\begin{tabular}{|c|c|c|c|c|c|c|c|c|c|c|}
\hline \multirow[t]{2}{*}{ Variant } & \multirow[t]{2}{*}{ Symbol } & \multicolumn{4}{|c|}{ N1=100 kg/ha N s.a. } & \multicolumn{4}{|c|}{$\mathrm{N} 2=50 \mathrm{~kg} / \mathrm{ha} \mathrm{N}$ s.a. } & \multirow{2}{*}{$\begin{array}{c}\text { Diff } \\
\text { Protein } \\
(\%) \\
\text { N1-N2 }\end{array}$} \\
\hline & & Protein & $(\%)$ & $\begin{array}{l}\text { Diff. } \\
\text { S1\% }\end{array}$ & Signif & Protein & $(\%)$ & $\begin{array}{l}\text { Diff. } \\
\text { S1\% }\end{array}$ & Signif & \\
\hline ARIESAN & S1 & 11.47 & 100.0 & 0.00 & Mt. & 9.80 & 100.0 & 0.00 & Mt. & 1,67 \\
\hline APULLUM & S2 & 10.13 & 88.4 & -1.33 & 000 & 9.10 & 92.9 & -0.70 & 00 & 1,03 \\
\hline TURDA 2000 & S3 & 10.40 & 90.7 & -1.07 & 000 & 9.77 & 99.7 & -0.03 & - & 0,63 \\
\hline DUMBRAVA & S4 & 9.27 & 80.8 & -2.20 & 000 & 8.57 & 87.4 & -1.23 & 000 & 0,70 \\
\hline ANDRADA & S5 & 10.37 & 90.4 & -1.10 & 000 & 8.90 & 90.8 & -0.90 & 000 & 1,47 \\
\hline DELABRAD & S6 & 10.23 & 89.2 & -1.23 & 000 & 9.80 & 100.0 & 0.00 & - & 0,43 \\
\hline FAUR & S7 & 9.63 & 84.0 & -1.83 & 000 & 9.13 & 93.2 & -0.67 & 0 & 0,50 \\
\hline GLOSA & S8 & 10.93 & 95.3 & -0.53 & 0 & 9.07 & 92.5 & -0.73 & 00 & 1,87 \\
\hline MIRANDA & S9 & 10.13 & 88.4 & -1.33 & 000 & 9.03 & 92.2 & -0.77 & 00 & 1,10 \\
\hline PITAR & S10 & 10.60 & 92.4 & -0.87 & 00 & 9.73 & 99.3 & -0.07 & - & 0,87 \\
\hline JOSEF & S11 & 10.77 & 93.9 & -0.70 & 00 & 10.03 & 102.4 & 0.23 & - & 0,73 \\
\hline RENAN & S12 & 10.77 & 93.9 & -0.70 & 00 & 10.17 & 103.7 & 0.37 & - & 0,60 \\
\hline CAPO & S13 & 10.57 & 92.2 & -0.90 & 000 & 9.43 & 96.3 & -0.37 & - & 1,13 \\
\hline APACHE & S14 & 9.27 & 80.8 & -2.20 & 000 & 8.77 & 89.5 & -1.03 & 000 & 0,50 \\
\hline ARLEQUIN & S15 & 8.87 & 77.3 & -2.60 & 000 & 8.80 & 89.8 & -1.00 & 000 & 0,07 \\
\hline EXOTIC & S16 & 9.87 & 86.0 & -1.60 & 000 & 9.70 & 99.0 & -0.10 & - & 0,17 \\
\hline MV. KOLO & S17 & 11.20 & 97.7 & -0.27 & - & 10.20 & 104.1 & 0.40 & - & 1,00 \\
\hline CRISTINA & S18 & 11.10 & 96.8 & -0.37 & - & 10.20 & 104.1 & 0.40 & - & 0,90 \\
\hline ELEMENT & S19 & 9.90 & 86.3 & -1.57 & 000 & 10.13 & 103.4 & 0.33 & - & 0,23 \\
\hline BERES & S20 & 10.90 & 95.1 & -0.57 & 0 & 9.80 & 100.0 & 0.00 & - & 1,10 \\
\hline GALIO & S21 & 9.70 & 84.6 & -1.77 & 000 & 9.47 & 96.6 & -0.33 & - & 0,23 \\
\hline FULVIO & S22 & 10.17 & 88.7 & -1.30 & 000 & 10.20 & 104.1 & 0.40 & - & 0,03 \\
\hline MAGISTRAL & S23 & 9.47 & 82.6 & -2.00 & 000 & 9.20 & 93.9 & -0.60 & 0 & 0,27 \\
\hline LOVRIN 34 & S24 & 10.10 & 88.1 & -1.37 & 000 & 9.20 & 93.9 & -0.60 & 0 & 0,90 \\
\hline CRIȘANA & S25 & 9.93 & 86.6 & -1.53 & 000 & 9.50 & 96.9 & -0.30 & - & 0,43 \\
\hline
\end{tabular}

Tab. 9. Analysis of variance and F sample for the polifactorial experience $(2 \times 25)$ with winter wheat varieties of for wet gluten (\%), at ARDS Turda, 2015

\begin{tabular}{cccccc}
\hline Variation source & Square sum & Liberty degrees & Mean Square & $\mathrm{F}$ & Signif. \\
\hline Fertilization (F) & 50,344 & 1 & 50,344 & 58,264 & $* * *$ \\
\hline Genotipe (G) & 242,609 & 24 & 10,109 & 56,203 & $* * *$ \\
\hline F x G & 29,810 & 24 & 1,242 & 6,906 & $* * *$ \\
\hline Repetions (R) & 0,965 & 2 & 0,483 & & \\
\hline FxR & 1,728 & 2 & 0,864 & \\
\hline GxR & 6,814 & 48 & 0,141 & \\
\hline FxGxR & 10,451 & 48 & 0,217 & \\
\hline Erorr F & 1,728 & 2 & 0,864 & \\
\hline Erorr G & 17,266 & 96 & 0,179 & \\
\hline Total & 342,724 & 149 & & \\
\hline
\end{tabular}

Tab.10. Influence of fertilization on wet gluten content for winter wheat varieties at ARDS Turda, 2015

\begin{tabular}{|c|c|c|c|c|c|c|}
\hline Variant & Symbol & Wet gluten & $(\%)$ & $\begin{array}{c}\text { Diff. to } \mathrm{N}_{1} \\
(\%)\end{array}$ & Signif. & Duncan \\
\hline $\mathbf{N}_{100} \mathbf{P}_{50}$ & $\mathrm{~F}_{1}$ & 19,69 & 100,0 & 0,00 & Mt. & $\mathrm{a}$ \\
\hline $\mathbf{N}_{50} \mathbf{P}_{50}$ & $\mathrm{~F}_{2}$ & 18,53 & 94,1 & $-1,16$ & 0 & $\mathrm{~b}$ \\
\hline DL p 5\% & \multicolumn{2}{|c|}{ DS 5\% } & & 0,65 & & \multirow{3}{*}{0,65} \\
\hline DL p 1\% & & & & 1,51 & & \\
\hline DL p $0,1 \%$ & & & & 4,80 & & \\
\hline
\end{tabular}


Tab. 11. The influence of genotype to wet gluten content (\%) for winter wheat varieties at ARDS Turda, 2015

\begin{tabular}{|c|c|c|c|c|c|c|}
\hline Variant & Symbol & $\begin{array}{c}\text { Wet } \\
\text { gluten }\end{array}$ & $(\%)$ & Diff. to $S_{1}(\%)$ & Signif. & Duncan \\
\hline ARIESAN & $\mathrm{S}_{1}$ & 20,67 & 100,0 & 0,00 & Mt. & $\mathrm{ab}$ \\
\hline APULLUM & $\mathrm{S}_{2}$ & 18,65 & 90,2 & $-2,02$ & 000 & $\mathrm{~h}$ \\
\hline TURDA 2000 & $\mathrm{~S}_{3}$ & 19,38 & 93,8 & $-1,28$ & 000 & ef \\
\hline DUMBRAVA & $\mathrm{S}_{4}$ & 16,50 & 79,8 & $-4,17$ & 000 & $\mathrm{k}$ \\
\hline ANDRADA & $\mathrm{S}_{5}$ & 18,55 & 89,8 & $-2,12$ & 000 & $\mathrm{~h}$ \\
\hline DELABRAD & $\mathrm{S}_{6}$ & 19,37 & 93,7 & $-1,30$ & 000 & ef \\
\hline FAUR & $\mathrm{S}_{7}$ & 18,37 & 88,9 & $-2,30$ & 000 & hi \\
\hline GLOSA & $\mathrm{S}_{8}$ & 19,85 & 96,0 & $-0,82$ & 00 & cde \\
\hline MIRANDA & $\mathrm{S}_{9}$ & 17,98 & 87,0 & $-2,68$ & 000 & $\mathrm{ij}$ \\
\hline PITAR & $\mathrm{S}_{10}$ & 19,43 & 94,0 & $-1,23$ & 000 & ef \\
\hline JOSEF & $\mathrm{S}_{11}$ & 20,62 & 99,8 & $-0,05$ & - & $\mathrm{b}$ \\
\hline RENAN & $\mathrm{S}_{12}$ & 20,35 & 98,5 & $-0,32$ & - & $\mathrm{bc}$ \\
\hline CAPO & $\mathrm{S}_{13}$ & 20,03 & 96,9 & $-0,63$ & 0 & $\mathrm{~cd}$ \\
\hline APACHE & $\mathrm{S}_{14}$ & 17,50 & 84,7 & $-3,17$ & 000 & $\mathrm{j}$ \\
\hline ARLEQUIN & $\mathrm{S}_{15}$ & 16,33 & 79,0 & $-4,33$ & 000 & $\mathrm{k}$ \\
\hline EXOTIC & $\mathrm{S}_{16}$ & 18,63 & 90,2 & $-2,03$ & 000 & $\mathrm{~h}$ \\
\hline MV. KOLO & $\mathrm{S}_{17}$ & 20,75 & 100,4 & 0,08 & - & $a b$ \\
\hline CRISTINA & $\mathrm{S}_{18}$ & 20,78 & 100,6 & 0,12 & - & $a b$ \\
\hline ELEMENT & $\mathrm{S}_{19}$ & 19,30 & 93,4 & $-1,37$ & 000 & $\mathrm{fg}$ \\
\hline BERES & $\mathrm{S}_{20}$ & 21,18 & 102,5 & 0,52 & $*$ & $\mathrm{a}$ \\
\hline GALIO & $\mathrm{S}_{21}$ & 18,83 & 91,1 & $-1,83$ & 000 & gh \\
\hline FULVIO & $\mathrm{S}_{22}$ & 19,80 & 95,8 & $-0,87$ & 000 & def \\
\hline MAGISTRAL & $\mathrm{S}_{23}$ & 17,82 & 86,2 & $-2,85$ & 000 & $\mathrm{j}$ \\
\hline LOVRIN 34 & $\mathrm{~S}_{24}$ & 18,62 & 90,1 & $-2,05$ & 000 & $\mathrm{~h}$ \\
\hline CRIȘANA & $\mathrm{S}_{25}$ & 18,35 & 88,8 & $-2,32$ & 000 & hi \\
\hline DL p 5\% & DS 5\% & & & 0,49 & & \\
\hline DL p 1\% & & & & 0,64 & & $0,49-0,60$ \\
\hline DL p $0,1 \%$ & & & & 0,83 & & \\
\hline
\end{tabular}

Tab.12. Influence of fertilization on wet gluten (\%) for winter wheat varieties at ARDS Turda, 2015

\begin{tabular}{|c|c|c|c|c|c|c|c|c|c|c|}
\hline \multirow[b]{2}{*}{ Variant } & \multirow[b]{2}{*}{ Symbol } & \multicolumn{4}{|c|}{$\mathrm{N}_{1}=100 \mathrm{~kg} / \mathrm{ha} N$ s.a. } & \multicolumn{4}{|c|}{$\mathrm{N}_{2}=50 \mathrm{~kg} / \mathrm{ha} \mathrm{N}$ s.a. } & \multirow[b]{2}{*}{$\begin{array}{l}\text { Diff. Wet } \\
\text { gluten } \\
\text { (\%) N1-N2 }\end{array}$} \\
\hline & & $\begin{array}{l}\text { Wet } \\
\text { gluten }\end{array}$ & $(\%)$ & $\begin{array}{c}\text { Diff. } \\
\text { S1 } \\
\%\end{array}$ & Signif. & $\begin{array}{l}\text { Wet } \\
\text { gluten }\end{array}$ & $(\%)$ & $\begin{array}{c}\text { Diff. } \\
\text { S1 } \\
\%\end{array}$ & Signif. & \\
\hline ARIESAN & $\mathrm{S}_{1}$ & 22.57 & 100.0 & 0.00 & Mt. & 18.77 & 100.0 & 0.00 & Mt. & 3.80 \\
\hline APULLUM & $\mathrm{S}_{2}$ & 19.23 & 85.2 & -3.33 & 000 & 18.07 & 96.3 & -0.70 & 0 & 1.17 \\
\hline TURDA 2000 & $\mathrm{~S}_{3}$ & 20.00 & 88.6 & -2.57 & 000 & 18.77 & 100.0 & 0.00 & - & 1.23 \\
\hline DUMBRAVA & $\mathrm{S}_{4}$ & 16.93 & 75.0 & -5.63 & 000 & 16.07 & 85.6 & -2.70 & 000 & 0.87 \\
\hline ANDRADA & $\mathrm{S}_{5}$ & 19.47 & 86.3 & -3.10 & 000 & 17.63 & 94.0 & -1.13 & 00 & 1.83 \\
\hline DELABRAD & $\mathrm{S}_{6}$ & 19.67 & 87.1 & -2.90 & 000 & 19.07 & 101.6 & 0.30 & - & 0.60 \\
\hline FAUR & $\mathrm{S}_{7}$ & 18.63 & 82.6 & -3.93 & 000 & 18.10 & 96.4 & -0.67 & - & 0.53 \\
\hline GLOSA & $\mathrm{S}_{8}$ & 21.20 & 93.9 & -1.37 & 000 & 18.50 & 98.6 & -0.27 & - & 2.70 \\
\hline MIRANDA & $\mathrm{S}_{9}$ & 19.30 & 85.5 & -3.27 & 000 & 16.67 & 88.8 & -2.10 & 000 & 2.63 \\
\hline PITAR & $\mathrm{S}_{10}$ & 20.10 & 89.1 & -2.47 & 000 & 18.77 & 100.0 & 0.00 & - & 1.33 \\
\hline JOSEF & $\mathrm{S}_{11}$ & 21.13 & 93.6 & -1.43 & 000 & 20.10 & 107.1 & 1.33 & $* * *$ & 1.03 \\
\hline RENAN & $\mathrm{S}_{12}$ & 21.13 & 93.6 & -1.43 & 000 & 19.57 & 104.3 & 0.80 & $*$ & 1.57 \\
\hline CAPO & $\mathrm{S}_{13}$ & 20.43 & 90.5 & -2.13 & 000 & 19.63 & 104.6 & 0.87 & $*$ & 0.80 \\
\hline APACHE & $\mathrm{S}_{14}$ & 17.63 & 78.1 & -4.93 & 000 & 17.37 & 92.5 & -1.40 & 000 & 0.27 \\
\hline ARLEQUIN & $\mathrm{S}_{15}$ & 16.67 & 73.9 & -5.90 & 000 & 16.00 & 85.3 & -2.77 & 000 & 0.67 \\
\hline EXOTIC & $\mathrm{S}_{16}$ & 18.87 & 83.6 & -3.70 & 000 & 18.40 & 98.0 & -0.37 & - & 0.47 \\
\hline MV. KOLO & $\mathrm{S}_{17}$ & 21.90 & 97.0 & -0.67 & - & 19.60 & 104.4 & 0.83 & $*$ & 2.30 \\
\hline CRISTINA & $\mathrm{S}_{18}$ & 21.57 & 95.6 & -1.00 & 00 & 20.00 & 106.6 & 1.23 & $* * *$ & 1.57 \\
\hline ELEMENT & $\mathrm{S}_{19}$ & 19.27 & 85.4 & -3.30 & 000 & 19.33 & 103.0 & 0.57 & - & 0.07 \\
\hline BERES & $\mathrm{S}_{20}$ & 21.53 & 95.4 & -1.03 & 00 & 20.83 & 111.0 & 2.07 & $* * *$ & 0.70 \\
\hline
\end{tabular}


Tab.12. (continued) Influence of fertilization on wet gluten (\%) for winter wheat varieties at ARDS Turda, 2015

\begin{tabular}{|c|c|c|c|c|c|c|c|c|c|c|}
\hline \multirow[b]{2}{*}{ Variant } & \multirow[b]{2}{*}{ Symbol } & \multicolumn{4}{|c|}{$\mathrm{N}_{1}=100 \mathrm{~kg} / \mathrm{ha} \mathrm{N}$ s.a. } & \multicolumn{4}{|c|}{$\mathrm{N}_{2}=50 \mathrm{~kg} / \mathrm{ha} \mathrm{N}$ s.a. } & \multirow[b]{2}{*}{$\begin{array}{c}\text { Diff. Wet } \\
\text { gluten } \\
\text { (\%) N1-N2 }\end{array}$} \\
\hline & & $\begin{array}{c}\text { Wet } \\
\text { gluten }\end{array}$ & $(\%)$ & $\begin{array}{l}\text { Diff. } \\
\text { S1 } \\
\%\end{array}$ & Signif. & $\begin{array}{c}\text { Wet } \\
\text { gluten }\end{array}$ & $(\%)$ & $\begin{array}{l}\text { Diff. } \\
\text { S1 } \\
\%\end{array}$ & Signif. & \\
\hline GALIO & $S_{21}$ & 18.97 & 84.0 & $\frac{10}{-3.60}$ & 000 & 18.70 & 99.6 & -0.07 & - & 0.27 \\
\hline FULVIO & $S_{22}$ & 19.90 & 88.2 & -2.67 & 000 & 19.70 & 105.0 & 0.93 & ** & 0.20 \\
\hline MAGISTRAL & $S_{23}$ & 18.27 & 80.9 & -4.30 & 000 & 17.37 & 92.5 & -1.40 & 000 & 0.90 \\
\hline LOVRIN 34 & $\mathrm{~S}_{24}$ & 18.97 & 84.0 & -3.60 & 000 & 18.27 & 97.3 & -0.50 & - & 0.70 \\
\hline CRIȘANA & $\mathrm{S}_{25}$ & 18.80 & 83.3 & -3.77 & 000 & 17.90 & 95.4 & -0.87 & 0 & 0.90 \\
\hline \multicolumn{11}{|c|}{$-0,69 ;$ DL $1 \%-0,91$} \\
\hline
\end{tabular}

Tab. 13. Analysis of variance and $\mathrm{F}$ sample for the polifactorial experience $(2 \times 25)$ with winter wheat varieties of for Zeleny index (\%), at ARDS Turda, 2015

\begin{tabular}{lccccc}
\hline Variation source & Square sum & Liberty degrees & Mean Square & F & Signif. \\
\hline Fertilization $(\mathrm{F})$ & 820,872 & 1 & 820,872 & 73,947 & $* * *$ \\
\hline Genotipe $(\mathrm{G})$ & 2769,065 & 24 & 115,377 & 70,213 & $* * *$ \\
\hline F x G & 531,579 & 24 & 22,149 & 13,479 & $* * *$ \\
\hline Repetions (R) & 9,000 & 2 & 4,500 & & \\
\hline FxR & 22,201 & 2 & 11,101 & & \\
\hline GxR & 46,569 & 48 & 0,970 & \\
\hline FxGxR & 111,181 & 48 & 2,316 & \\
\hline Erorr F & 22,201 & 2 & 11,101 & \\
\hline Erorr G & 157,751 & 96 & 1,643 & \\
\hline Total & 4310,470 & 149 & & & \\
\hline
\end{tabular}

Tab.14. Influence of fertilization on Zeleny index for winter wheat varieties at ARDS Turda, 2015

\begin{tabular}{|c|c|c|c|c|c|c|}
\hline Variant & Symbol & $\begin{array}{c}\text { Zeleny } \\
\text { (ml) }\end{array}$ & $(\%)$ & $\begin{array}{c}\text { Diff. to } N_{1} \\
(\%)\end{array}$ & Signif. & Duncan \\
\hline $\mathbf{N}_{100} \mathbf{P}_{50}$ & $\mathrm{~F}_{1}$ & 27,27 & 100,0 & 0,00 & Mt. & $a$ \\
\hline $\mathbf{N}_{50} \mathbf{P}_{50}$ & $\mathrm{~F}_{2}$ & 22,59 & 82,8 & $-12,71$ & 0 & $\mathrm{~b}$ \\
\hline & DL p 5\% & DS & & 2,34 & & 2,34 \\
\hline & \multirow{2}{*}{\multicolumn{2}{|c|}{ DL p 1\% }} & & 5,40 & & \\
\hline & & & & 17,19 & & \\
\hline
\end{tabular}

The results obtained from ARDS Turda, 2015 us to say that after assessing the quality of wet gluten content is more objective than the one that is done after protein content. Some varieties can accumulate a higher protein content, but they are not quality.

Ariesan variety was the highest variation of wet gluten content in variant $\mathrm{N} 1(100 \mathrm{~kg} /$ ha $\mathrm{N}$ active substance) to $\mathrm{N} 2(50 \mathrm{~kg} / \mathrm{ha} \mathrm{N}$ active substance), which is $3.80 \%$ (Tab. 12).

Sedimentation method, developed by Zeleny is simple and rapid and is based on the swelling of gluten and sedimentation of the particles in acid medium. Stability Index shows a relatively high sedimentation in different experimental conditions and is less influenced by environment.

As can be seen from Tab. 13 analysis of variance forZelenyindex, itisconditionedinahigh proportion of genotype (SP $=2769.065)$, when referring to the sum of squares attributed to this factor, but it can be improved through fertilization $\left(\mathrm{F}=73.947^{* *}\right)$.

By increasing the dose of nitrogen to obtain an increase in sedimentation index for winter wheat varieties in 2015 with $4.68 \mathrm{ml}$ on variant N1 (100 $\mathrm{kg} /$ ha $\mathrm{N}$ active substance) to $\mathrm{N} 2$ (50 kg/ha $\mathrm{N}$ active substance) as is apparent from the data presented in Tab. 14. 
Tab. 15. The influence of genotype to Zeleny index (\%) for winter wheat varieties at ARDS Turda, 2015

\begin{tabular}{|c|c|c|c|c|c|c|}
\hline Variant & Symbol & Zeleny (ml) & (\%) & Diff to $S_{1}(\%)$ & Signif. & Duncan \\
\hline ARIESAN & $\mathrm{S}_{1}$ & 30.12 & 100.0 & 0.00 & Mt. & $\mathrm{bc}$ \\
\hline APULLUM & $\mathrm{S}_{2}$ & 25.40 & 84.3 & -4.72 & 000 & gh \\
\hline TURDA 2000 & $\mathrm{~S}_{3}$ & 29.58 & 98.2 & -0.53 & - & $\mathrm{cd}$ \\
\hline DUMBRAVA & $\mathrm{S}_{4}$ & 19.70 & 65.4 & -10.42 & 000 & $\mathrm{k}$ \\
\hline ANDRADA & $\mathrm{S}_{5}$ & 23.17 & 76.9 & -6.95 & 000 & $\mathrm{i}$ \\
\hline DELABRAD & $\mathrm{S}_{6}$ & 25.10 & 83.3 & -5.02 & 000 & gh \\
\hline FAUR & $\mathrm{S}_{7}$ & 24.65 & 91.8 & -5.47 & 000 & $\mathrm{~h}$ \\
\hline GLOSA & $\mathrm{S}_{8}$ & 26.65 & 88.5 & -3.47 & 000 & $\mathrm{fg}$ \\
\hline MIRANDA & $\mathrm{S}_{9}$ & 19.92 & 66.1 & -10.20 & 000 & $\mathrm{k}$ \\
\hline PITAR & $\mathrm{S}_{10}$ & 24.97 & 82.9 & -5.15 & 000 & gh \\
\hline JOSEF & $\mathrm{S}_{11}$ & 30.30 & 100.6 & 0.18 & - & $\mathrm{bc}$ \\
\hline RENAN & $\mathrm{S}_{12}$ & 26.55 & 88.2 & -3.57 & 000 & fg \\
\hline CAPO & $\mathrm{S}_{13}$ & 27.42 & 91.0 & -2.70 & 000 & ef \\
\hline APACHE & $\mathrm{S}_{14}$ & 19.27 & 64.0 & -10.85 & 000 & $\mathrm{k}$ \\
\hline ARLEQUIN & $\mathrm{S}_{15}$ & 15.87 & 52.7 & -14.25 & 000 & l \\
\hline EXOTIC & $\mathrm{S}_{16}$ & 22.97 & 76.3 & -7.15 & 000 & $\mathrm{i}$ \\
\hline MV. KOLO & $\mathrm{S}_{17}$ & 31.43 & 104.4 & 1.32 & - & $\mathrm{b}$ \\
\hline CRISTINA & $\mathrm{S}_{18}$ & 28.33 & 94.1 & -1.78 & 0 & de \\
\hline ELEMENT & $\mathrm{S}_{19}$ & 25.08 & 83.3 & -5.03 & 000 & gh \\
\hline BERES & $\mathrm{S}_{20}$ & 33.05 & 109.7 & 2.93 & *** & $\mathrm{a}$ \\
\hline GALIO & $\mathrm{S}_{21}$ & 24.67 & 81.9 & -5.45 & 000 & $\mathrm{~h}$ \\
\hline FULVIO & $\mathrm{S}_{22}$ & 25.50 & 84.7 & -4.62 & 000 & $\mathrm{gh}$ \\
\hline MAGISTRAL & $\mathrm{S}_{23}$ & 16.82 & 55.8 & -13.30 & 000 & l \\
\hline LOVRIN 34 & $\mathrm{~S}_{24}$ & 25.37 & 84.2 & -4.75 & 000 & gh \\
\hline CRIȘANA & $\mathrm{S}_{25}$ & 21.45 & 71.2 & -8.67 & 000 & $\mathrm{j}$ \\
\hline \multicolumn{6}{|c|}{ DL p 5\% DS 5\% 1,47 DL p 1\% 1,95 DL p 0,1\% 2,51 } & $1,47-1,83$ \\
\hline
\end{tabular}

The data in Tab. 15 is that the variety Beres obtained the highest Zeleny index (sedimentation index) values 33,05 \%, and Arieșan is not far from it $30,12 \%$.

The results are particularly important because emphasizes the importance of nitrogen fertilization to obtain yields with good quality indices, as in the case of sedimentation index. Of course there are varieties such as: Apache, Arlequin and Magistral that do not change regardless of the quality by the level of nitrogen fertilization, meaning that they are endowed with a potential low quality, genetically (Tab. 16).

\section{CONCLUSION}

The result of research is to test the response to fertilization of winter wheat variety released in production, and behavior evaluation for Romanian winter wheat varieties compared to foreign ones.

To the 25 winter wheat varieties studied in at ARDS Turda result genetic differences in terms of production potential, indicated by the analysis of variance; and the highest level of production being obtained by the cultivars Apache (France) Dumbrava (Romania) Exotic (Germany).

Winter wheat genotypes react differently to fertilization, the yield increases between 403 and $1565 \mathrm{~kg} / \mathrm{ha}$, noting Dumbrava variety among them which were obtained from $10060 \mathrm{~kg} / \mathrm{ha}$ in variant $\mathrm{N} 1100 \mathrm{~kg} / \mathrm{ha} \mathrm{N}$ active substance and $8778 \mathrm{~kg} / \mathrm{ha}$ in variant $\mathrm{N} 2$ to $50 \mathrm{~kg} / \mathrm{ha} \mathrm{N}$ active substance.

Fertilization, especially with nitrogen contribute significantly to achieving good quality for grain, containing appropriate protein for baking industry. 
Tab. 16. Influence of fertilization on Zeleny index (\%) for winter wheat varieties at ARDS Turda, 2015

\begin{tabular}{|c|c|c|c|c|c|c|c|c|c|c|}
\hline \multirow[b]{2}{*}{ Variant } & \multirow[b]{2}{*}{ Symbol } & \multicolumn{4}{|c|}{$\mathrm{N}_{1}=100 \mathrm{~kg} / \mathrm{ha} \mathrm{N}$ s.a. } & \multicolumn{4}{|c|}{$\mathrm{N}_{2}=50 \mathrm{~kg} /$ ha N s.a. } & \multirow{2}{*}{$\begin{array}{c}\text { Diff } \\
\text { Zeleny } \\
(\mathrm{ml}) \\
\mathrm{N} 1-\mathrm{N} 2\end{array}$} \\
\hline & & $\begin{array}{l}\text { Zeleny } \\
(\mathrm{ml})\end{array}$ & $(\%)$ & $\begin{array}{c}\text { Dif. S1 } \\
\%\end{array}$ & Signif & $\begin{array}{l}\text { Zeleny } \\
(\mathrm{ml})\end{array}$ & $(\%)$ & $\begin{array}{c}\text { Dif. } \\
\text { S1\% }\end{array}$ & Signif & \\
\hline ARIESAN & $\mathrm{S}_{1}$ & 34.83 & 100.0 & 0.00 & Mt. & 25.40 & 100.0 & 0.00 & Mt. & 9.43 \\
\hline APULLUM & $\mathrm{S}_{2}$ & 26.30 & 75.5 & -8.53 & 000 & 24.50 & 96.5 & -0.90 & - & 1.80 \\
\hline TURDA 2000 & $\mathrm{~S}_{3}$ & 30.53 & 87.7 & -4.30 & 000 & 28.63 & 112.7 & 3.23 & $* *$ & 1.90 \\
\hline DUMBRAVA & $\mathrm{S}_{4}$ & 25.17 & 72.2 & -9.67 & 000 & 14.23 & 56.0 & -11.17 & 000 & 10.93 \\
\hline ANDRADA & $\mathrm{S}_{5}$ & 26.33 & 75.6 & -8.50 & 000 & 20.00 & 78.7 & -5.40 & 000 & 6.33 \\
\hline DELABRAD & $\mathrm{S}_{6}$ & 27.07 & 77.7 & -7.77 & 000 & 23.13 & 91.1 & -2.27 & 0 & 3.93 \\
\hline FAUR & $\mathrm{S}_{7}$ & 22.87 & 65.6 & -11.97 & 000 & 26.43 & 104.1 & 1.03 & - & 3.57 \\
\hline GLOSA & $\mathrm{S}_{8}$ & 31.37 & 90.0 & -3.47 & 00 & 21.93 & 86.4 & -3.47 & 00 & 9.43 \\
\hline MIRANDA & $\mathrm{S}_{9}$ & 23.60 & 67.8 & -11.23 & 000 & 16.23 & 63.9 & -9.17 & 000 & 7.37 \\
\hline PITAR & $\mathrm{S}_{10}$ & 28.30 & 81.2 & -6.53 & 000 & 21.63 & 85.2 & -3.77 & 000 & 6.67 \\
\hline JOSEF & $\mathrm{S}_{11}$ & 32.83 & 94.3 & -2.00 & - & 27.77 & 109.3 & 2.37 & $*$ & 5.07 \\
\hline RENAN & $\mathrm{S}_{12}$ & 30.97 & 88.9 & -3.87 & 000 & 22.13 & 87.1 & -3.27 & 00 & 8.83 \\
\hline CAPO & $\mathrm{S}_{13}$ & 28.40 & 81.5 & -6.43 & 000 & 26.43 & 104.1 & 1.03 & - & 1.97 \\
\hline APACHE & $\mathrm{S}_{14}$ & 19.70 & 56.6 & -15.13 & 000 & 18.83 & 74.1 & -6.57 & 000 & 0.87 \\
\hline ARLEQUIN & $\mathrm{S}_{15}$ & 18.30 & 52.5 & -16.53 & 000 & 13.43 & 52.9 & -11.97 & 000 & 4.87 \\
\hline EXOTIC & $\mathrm{S}_{16}$ & 25.00 & 71.8 & -9.83 & 000 & 20.93 & 82.4 & -4.47 & 000 & 4.07 \\
\hline MV. KOLO & $\mathrm{S}_{17}$ & 37.50 & 107.7 & 2.67 & $*$ & 25.37 & 99.9 & -0.03 & - & 12.13 \\
\hline CRISTINA & $\mathrm{S}_{18}$ & 31.93 & 91.7 & -2.90 & 00 & 24.73 & 97.4 & -0.67 & - & 7.20 \\
\hline ELEMENT & $\mathrm{S}_{19}$ & 26.53 & 76.2 & -8.30 & 000 & 23.63 & 93.0 & -1.77 & - & 2.90 \\
\hline BERES & $\mathrm{S}_{20}$ & 33.03 & 94.8 & -1.80 & - & 33.07 & 130.2 & 7.67 & $* * *$ & 0.03 \\
\hline GALIO & $\mathrm{S}_{21}$ & 25.17 & 72.2 & -9.67 & 000 & 24.17 & 95.1 & -1.23 & - & 1.00 \\
\hline FULVIO & $\mathrm{S}_{22}$ & 25.93 & 74.4 & -8.90 & 000 & 25.07 & 98.7 & -0.33 & - & 0.87 \\
\hline MAGISTRAL & $\mathrm{S}_{23}$ & 17.63 & 50.6 & -17.20 & 000 & 16.00 & 63.0 & -9.40 & 000 & 1.63 \\
\hline LOVRIN 34 & $\mathrm{~S}_{24}$ & 27.40 & 78.7 & -7.43 & 000 & 23.33 & 91.9 & -2.07 & - & 4.07 \\
\hline CRIŞANA & $\mathrm{S}_{25}$ & 25.10 & 72.1 & -9.73 & 000 & 17.80 & 70.1 & -7.60 & 000 & 7.30 \\
\hline
\end{tabular}

The reaction to the fertilizer varieties of winter wheat tested in conditions of 2015 at ARDS Turda was different in terms of protein content, allowing identification of varieties very productive, as Arlequin which yielded low levels on both levels fertilization.

The results obtained from the variety Arieșan the protein content in 2015 averaged $11.47 \%$ in additional variant fertilized with nitrogen and $9.80 \%$ in the variant N50.

In most varieties, quality achieved in 2015, apreciated by the wet gluten content is modest to low, values over $22 \%$ they have not registered to any studied variety.

After wet gluten content obtained at fertilization variant with $100 \mathrm{~kg} /$ ha $\mathrm{N}$ active substance variety Arieșan ocupying the first place, yielding differences compared to him statistically being distinct and very significant negative.

The results obtained in Turda in terms of quality indices obtained, us to say that 2015 was not favorable from this point of view, because lower values were obtained in all varieties. explanations for this are the rains that occurred in July and that caused washing gluten and its qualitative impairment.

\section{REFERENCES}

1. Ceapoiu N, Bîlteanu G, Hera CR, Săulescu NN, Negulescu F, Bărbulescu A (1984). Grâul, Editura Academiei Republicii Socialiste România, București.

2. Ceapoiu N, Ittu G, Oproiu E (1974). Ereditatea conținutului şi calității proteinelor de grâu (Triticum aestivum ssp. 
vulgare), Probleme de genetică teoretică ssi aplicată, 6 (5):321-343

3. Ceclan OA, Racz I, Kadar R, Ceclan LA, Russu F, (2015). Influența nivelului de fertilizare asupra producției şi a unor indici calitativi la un set de soiuri de grâu de toamnă, AN. I.N.C.D.A. Fundulea, LXXXIII : 20-24.

4. Kadar R, Moldovan V, Tianu M, Marca V (1999). Cercetări privind calitatea de panificație a grâului de toamnă În: Contribuții ale cercetării științifice la Dezvoltarea agriculturii, Tipografia Boema Turda, VI:25-34.
5. Kadar R, Moldovan V, Moldovan M, Marca V, Tianu M (2002). Posibilități de realizare a progresului genetic în ameliorarea calității de panificație a grâului la S.C.A. Turda. Cercet. Genet. Veget. Anim., VII:71-87.

6. Kadar R (2002). Studiul interacțiunii genotip-condiții de cultură în realizarea calității de panificație la grâul de toamnă, Teză de doctorat, USAMV Cluj-Napoca.

7. Ionuț Racz, Ioan Haş, Vasile Moldovan, Rozalia Kadar, Adrian Ceclan (2014). Evaluarea stabilității producției şi a principalelor componente ale acesteia la un set de soiuri de grâu de toamnă, AN. I.N.C.D.A. Fundulea, LXXXII, 50-56. 